\title{
Survey of Context-Aware Video Transmission over Vehicular Ad-Hoc Networks (VANETs)
}

\author{
Reem F. Shaibani ${ }^{1, *}$, Ammar T. Zahary ${ }^{1}$ \\ ${ }^{1}$ Faculty of Computer and IT, Sana'a University, Sana’a, Yemen, reemfatwan@yahoo.com, aalzahary@gmail.com
}

\begin{abstract}
Vehicular ad-hoc networks (VANETs) are defined as a group of wireless mobile nodes that characterized by their high mobility. Therefore, the topology frequently changes and high communication overhead for exchanging a new topology information has been introduced. Due to the huge enrichment by the video data based on the quality of experience recognized by the driver and knowledge of the environment, video transmission over VANETs has been considered as a critical challenge. Context-aware vehicular ad-hoc network is a novel field that introduces developed solutions for the previous challenges in VANETs and guarantees the stability of its topology through different high mobility of nodes. In this paper, we investigated the general issues related to VANETs such as characteristics, applications, and current challenges. Furthermore, we reviewed the most significant relevant studies that apply a context-aware issue as a new technique in vehicular networks that helps to overcome VANETs challenges.
\end{abstract}

Keywords: Ad hoc network, Vehicular ad hoc network, VANET challenges, VANET characteristics, VANET applications, Video transmission over VANET, ad-hoc based routing for VANET, Wireless networks, Context-aware.

Received on 27 August 2018, accepted on 21 November 2018, published on 14 December 2018

Copyright (c) 2018 Reem F. Shaibani et al., licensed to EAI. This is an open access article distributed under the terms of the Creative Commons Attribution licence (http://creativecommons.org/licenses/by/3.0/), which permits unlimited use, distribution and reproduction in any medium so long as the original work is properly cited.

doi: 10.4108/eai.13-7-2018.156089

\section{Introduction}

Vehicular Ad-Hoc Networks (VANETs) have been considered an emerging technology due to the variety of their applications in Intelligent Transportation Systems (ITS). These networks have been used to describe the autonomous and self-organizing wireless communication network of mobile nodes which are mainly vehicles. By creating a vehicular network, each vehicle can exchange information to inform drivers in other vehicles about the current status of the traffic flow or the existence of a dangerous situation. Nodes in VANETs environment (vehicles) can communicate with each other as servers and/or clients for exchanging and sharing information [15].

These vehicles are more dynamic because are usually moving at a very high speed and changing their position constantly. Their high mobility also leads to a highly

*Corresponding author. Email: reemfatwan@yahoo.com dynamic topology while the link conditions between vehicles changes [6-10].

Although researchers have achieved much great progress on VANETs study, there are still some challenges that need to be investigated and solved such as,. communication, security, applications, stimulation, verification, services, etc. [8, 11]. By considering the characteristics of high dynamic topology and high mobility vehicles in VANETs, various challenges and constraints have involved with respect to communication issues such as a high link break, very high route discovery, and very low link life time between nodes [6, 12, 13].

Due to the main goal of VANETs which consists in improving road safety, convenience and comfort of the passengers in the vehicle [7, 14], there are a lot of research works have done in area of VANETs network communications which allow the development of a large number of applications and can provide a wide range of information to drivers and travellers. Collision warning, 
lane change/merge assistance, traffic management and emergency warnings are a few promising examples of VANET applications [7, 11, 15]. Significantly, the deploying of VANETs leads to enhance traffic safety and efficiency by reducing the traffic jams and accidents.

Many routing protocols for ad-hoc networks have been proposed to improve the routing performance and reliability. The up to date standardized protocols are classified into three categories [16] according to their functionality: proactive routing protocols such as Optimized Link State Routing (OLSR), reactive routing protocols such as Ad-hoc On Demand Distance Vector (AODV), Dynamic Source Routing (DSR), and Temporally Ordered Routing Algorithm (TORA), and hybrid routing protocols such as Geographic Routing Protocol (GRP).

In particular, video transmission has been considered as a big issue over vehicular ad-hoc networks, because of the huge enrichment by the video data of the quality of experience recognized by the driver and knowledge of the environment in many applications such as emergency video calls, road traffic conditions, tourist information, etc. Furthermore, due to the strict Quality of Service requirements of video transmission [9].

The new field of context-awareness, due to its potential to promote the VANET applications, have become attracted by many automobile industry professionals and researchers recently. It is a new technique (context-aware technique) that introduces developed solutions for challenges of VANETs by using contextual information [17-19]. This kind of information helps in describing significantly the situation of driving which is also referred as driving context information. The contextual information can be the position, direction, velocity, and acceleration of the vehicle, traffic and weather information. All this contextual information can be used by the routing protocols to increase their performance[17].

In this paper, we checked on the most critical significant studies to explore the general issues identified with VANETs. First, we highlighted the general characteristics related to vehicular networks. Next, VANETs applications have been classified in details according to their purpose into three main categories. Then, various challenges and constraints have been investigated with respect to different issues that ought to be contemplated to empower the usage of ad-hoc based routing in VANET. Finally, we investigated existing works, strategies and applications that have been recently developed by applying a context-aware issue in video transmission to overcome vehicular ad-hoc networks challenges.

\section{Vehicular ad-hoc networks overview}

\subsection{General characteristics}

Recently, the vehicular ad-hoc networks (VANETs) have attracted great interest in academic and industry research field because of their unique attractive features and challenging characteristics. In this section, we highlighted the general characteristics related to vehicular networks that should be taken into consideration to enable the implementation of highly efficient communication protocols for VANET networks. Theses challenging characteristics are mentioned as follow:

(i) High mobility $[6,12]$ : The vehicles in VANETs usually are moving at high speed. This makes it harder to predict a vehicle's position and to make protection of node privacy.

(ii) Highly dynamic topology [7, 12, 15, 20, 21]: Due to high mobility and random speed of vehicles, the position of node changes frequently. As a result of this, network topology in VANETs tends to change frequently. The topology formed by VANETs is always changing as vehicles are moving at high speed. On highways, vehicles are moving at the speed of $60-70 \mathrm{mph}(25 \mathrm{~m} / \mathrm{sec})$. If the radio range between two vehicles is $125 \mathrm{~m}$, then the link between the two vehicles would last at most $10 \mathrm{sec}$. The topology of VANETs changes because of the movement of vehicles at high speed. Suppose two vehicles are moving at the speed of $20 \mathrm{~m} / \mathrm{sec}$ and the radio range between them is $160 \mathrm{~m}$, then the link between the two vehicles will last $160 / 20=8 \mathrm{sec}$.

(iii) Predictable mobility patterns $[7,15,21]$ : The mobility pattern of vehicles in VANETs depends on traffic environment, roads structure, the speed of vehicles, driver's driving behaviour and so on. The vehicles don't move in a random way because they are constrained by road topology and layout. Also they are constrained by the requirement to obey road signs and traffic light and to respond to other moving vehicles leading to predictability in term of their mobility.

(iv) Sufficient energy [7, 12, 15]: The power in VANETs is not a critical challenge because vehicles have the ability to provide continuous power via the long life battery. Thus, it has enough computing power. It is helpful for effective communication and making routing decisions.

(v) On-board sensors [7, 12, 15, 20]: In VANETs, the vehicles are assumed to be equipped with a sufficient number of sensors and computational resources like the processors, large memory capacity, advanced antenna technology and global position system (GPS) to obtain information for routing purposes. Thus, the vehicles can easily sense accurate information regarding to the vehicles' current position, speed and direction. This can be 
done by on-board sensors for the sake of efficient communication and routing decisions.

(vi) Variable network density [7]: Based on the traffic density, the network density in VANETs can be very high in the case of a traffic jam, or very low as in suburban traffic.

(vii) Large scale network [7, 12]: VANETs can be applied on one city, several cities or countries. This means that network scale in VANETs is geographically unlimited.

The general characteristics of VANETs are summarized as shown in Figure.1

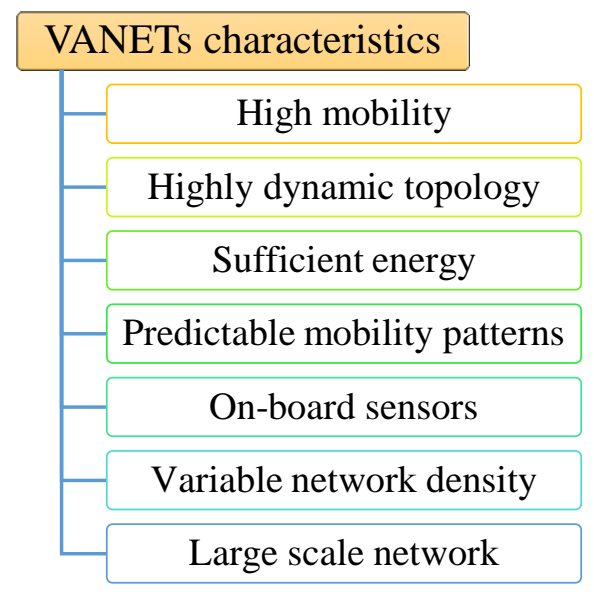

Figure 1. General characteristics of VANETs

\subsection{Classes of VANETs applications}

Safety and efficiency are two important requirements that should be considered to classify the applications over VANETs. As shown in figure 2., a classification of VANETs' applications is done by many studies according to their main purpose into three classes $[2,7,8,10,14$, $22,23]$ and can be summarized as follows:

\section{Traffic safety applications}

These applications represent the main motivation for developing VANETs which aim to improve driver/road safety and avoid accidents by enabling each vehicle to provide a warning in real time when a critical event is detected. The intention is to save people's lives and to provide a clean environment. Vehicle diagnostics and maintenance, curve speed warning, intersection collision avoidance, and pre-crash sensing are some of the use cases for traffic safety applications[2, 7, 8, 10, 14, 22, 23].

\section{Traffic efficiency applications:}

This category of applications aims to optimize the traffic flow of vehicles by decreasing the time taken by vehicle and situation of traffic jam. Efficiency and management applications also aim to optimize the routes while providing a reduction of fuel consumption and gas emission such as, enhanced route navigation/guidance and speed management applications[14, 22, 23].

\section{Infotainment applications (non-safety):}

These applications are referred to as entertainment or commercial applications. They aim to improve drivers and passengers comfort levels (make the driver's journey more pleasant) and to enhance traffic efficiency. They can provide drivers or passengers with information about weather and traffic, and detail the location of the nearest restaurant, petrol station or hotel and their prices. Infotainment applications can also provide services such as, media downloading and online games[2, 7, 8, 10, 14, $22,23]$.

\subsection{VANETs challenges}

Although researchers have achieved much great progress on VANETs' field, there are still some challenges that need to be investigated and solved. For example, communication, security, applications, stimulation, verification, services, etc.[8]. Thus, by considering the high dynamic topology and high mobility nodes of VANETs, VANETs have various challenges and constraints with respect to technical issues [6, 23] which are classified as follow:

- Network management: The network topology and channel condition can be changed rapidly because of high mobility of vehicles. So, it can't use structures like tree because these structures can't be set up and maintained when the topology is rapidly changed.

- Congestion and collision control: The variable network density create another challenge that effect on the control of collision and transmission errors. In rural and urban areas, the traffic load is low. In the other hand, the network segments frequently happen in rush hours and the traffic load is very high. Hence, the network congests and the collision occurs in it. In this case, it is necessary to design protocols for medium access control to avoid congestion and collision. 


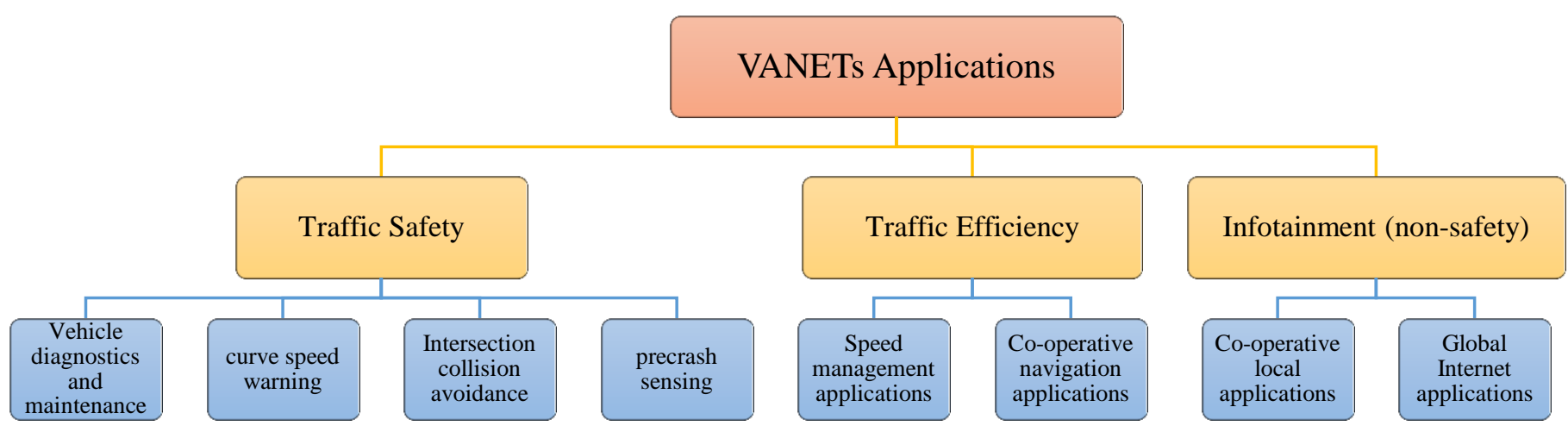

Figure 2. VANETs applications

- Environmental impact: In VANETs, the vehicles communicate by using the electromagnetic waves. This kind of waves is affected by the surrounded environment. Therefore, the environmental impact must be considered while deploying the VANETs.

- MAC design:. The MAC design is the key issue VANETs which this networks generally use the shared medium to communicate. In this case, there are many approaches have been given such as, TDMA, SDMA, and CSMA etc. In particular, IEEE 802.11 adopts the CSMA based Mac for VANETs.

- Security: Due to highly sensitive nature of information that is transformed over VANETs, the security of these information must be satisfied.

In other way, routing challenges in VANETs have been classified with respect to management of Quality of Service (QoS) for various services[16] as follow:

- Persistent topological changes because of high mobility of the vehicles.

- Variable network density and mobility of the vehicles on the road.

- Sparse distribution of vehicles in some geographical areas that leads to weak in connectivity and performance of the network.

- Efficient clustering and selection of Cluster Head $(\mathrm{CH})$ based on some predefined criteria.

- Intrusion detection and security.

Furthermore, according to communication between nodes, the challenges in VANETs can be classified into [10]:

(i) Security: The security of message content is a critical issue for vehicle to vehicle communication. The content of a received message has to be checked in a short time to be able to use the information as soon as possible.

(ii) Authentication: The authentication service is concerned with assuring that the communication is authentic in its entities. (iii) Integrity : The integrity service deals with the stability of a stream of messages.

(iv) Confidentiality: This service provides the confidentiality to the communication content and guarantees the privacy of drivers against unauthorized observers.

In [8], the key challenges are introduced as follow:

- Fundament limits and opportunities.

- Standards.

- Routing protocols.

- Connectivity.

- Cross-Layer.

- Cooperative Communication.

- Mobility.

- Security and Privacy.

- Validation.

According to previous studies, the various challenges of VANETs can be summarized as shown in figure 3 .

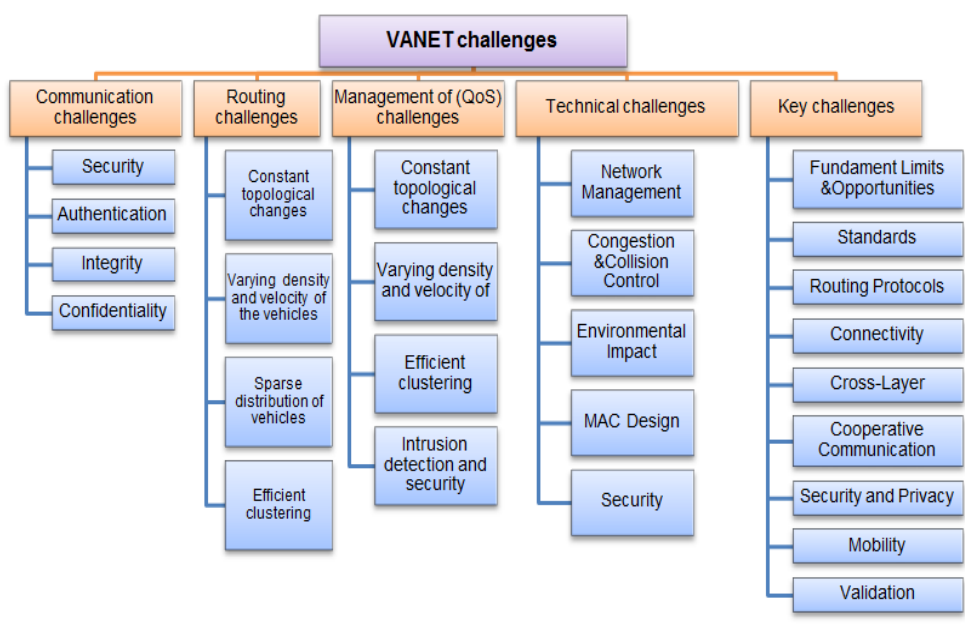

Figure 3. Challenges of VANETs 


\subsection{Ad-hoc based routing in VANETs}

Many routing protocols for ad-hoc networks have been proposed to improve the routing performance and reliability. The up to date standardized protocols are classified into three categories according to their functionality[16] as shown in Figure 4.:

- Proactive routing protocols.

- Reactive routing protocols.

- Hybrid routing protocols.

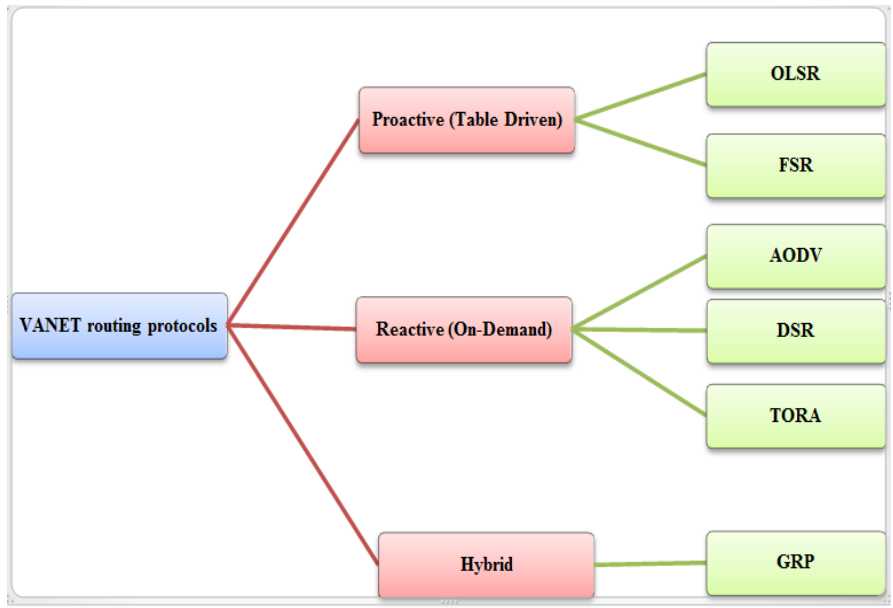

Figure 4. Ad-hoc based routing in VANETs

Proactive or table-driven routing protocols continuously monitor the topology changes of the network in order to have route information available between any source and destination at all time. All related routing information is periodically updated and stored in tables. This is due to the control messages that are broadcasted over the network even when the route is needed or not. As a result, there is a fast response to obtain routing information and it's easy to establish a session between nodes. On the other hand, there is a heavy traffic overhead according to the data that is kept by the nodes for route maintenance. Optimized Link State Routing (OLSR) is an example of a proactive routing protocols $[13,16]$.

Reactive or on-demand routing protocols establish the route for the source node to destination node only when there is a request to transmit data between them in the network. Thus, these protocols build routes when demanded. As a result, reactive routing protocols have bandwidth efficient which provides low control traffic and routing overhead. However, they offer high latency when discovering routes. The Ad-hoc On Demand Distance Vector (AODV), Dynamic Source Routing (DSR), and Temporally Ordered Routing Algorithm (TORA) are examples of reactive routing protocols $[13,16]$.

There are another kind of ad-hoc routing protocols with a combination of both reactive and proactive characteristics. These are referred to as hybrid routing protocols. They are used to provide more efficiency and scalability of the network. The main idea of hybrid routing protocols is the reducing a set of nodes which are sent over the network using the proactive routing approach for neighbour nodes that usually transmit data to far placed nodes. This availability of route for neighbour nodes guarantees no waste of bandwidth. Additionally, the flexibility and correctness of the reactive routing, and the overhead are greatly decreased by limitation of number of forwarding nodes. Geographic Routing Protocol (GRP) $[6,13,16]$ is an example of a hybrid routing protocols.

\section{Context-aware issue in VANETs}

In light of recent developments in information and communication technologies in the area of Intelligent Transportation Systems (ITS) specially in VANETs area, many researchers and industry professionals have become attracted to the new field which is called a context-aware vehicular ad-hoc networks. It is a new technique (contextaware technique) that introduces developed solutions to VANETs' challenges by using contextual information.

This kind of information helps in describing situation of the driving which is also referred to as driving context information. The context information can be the position, direction, velocity, and acceleration of the vehicle, traffic and weather information. All the context information can be used by the routing protocols to increase their performance[17].

According to the growth in the number of internet users and communication infrastructure to connect vehicles with internet, there is a need for the development of context-aware applications. There are many existing works, strategies and applications that have been recently developed by using a context-aware technique to overcome vehicular ad-hoc networks challenges. A brief descriptions of these works are given as follows:

Based on the analytical approaches in CAVE-NET, an overview of the recent achievements on two faces of context-aware vehicular networks have been introduced[24]. First, the modelling and prediction of macroscopic context information when vehicular traffic flow. Second, the use of the traffic flow as context information to deploy communication infrastructure in support of vehicular content distribution. They addressed the problem at two different levels:

(i) At the level of the vehicles, where the characteristics of vehicular flows and its impact on the information dissemination has been investigated.

(ii) At the level of the operators of the communication infrastructure, where the placement of RSUs as a function of vehicular flows has been considered. Therefore, they have considered both (V2V) and (I2V) communications.

The use of context-aware systems in researching has been proposed [17] to identify the convenient information to be provided to drivers without interrupting them while driving. In another way, to independent vehicles without providing them with non-important information, so decreasing the processing energy needed and improving 
the latency and quality of decisions made. In order to make the investigation more limited, the researchers in this work focused on the Quality of Information (QoI) to drivers and independent vehicles.

A unified architectural VANETs framework [25], which is called CARAVAN (Context-AwaRe Architecture for VANETs), has been presented[25]. The CARAVAN aims to integrate many VANETs concepts into a common framework and uses them on the dependency of the service requirements, connectivity properties and node mobility characteristics. The unified framework behaviour and adaptation is driven by contexts (context-aware approach). Contexts are related to service/application requirements, communication ability, mobility vectors and the mutual space-time relations of cars/nodes. The usage of contexts provides high level of adaptability and flexibility. In the CARAVAN, there are three layers have been defined: the mobility layer, the connectivity layer and the application layer. Such functional decomposition of the architecture provides ability to incremental modification of every layer via adding or via modifying layer internal components without the necessity of the redesign of other components of the architecture.

The extensive outdoor experiments [26] have showed the existing rate adaptation schemes for 802.11 wireless networks underutilize the link capacity in vehicular environments. The researchers designed, implemented and evaluated a novel Context-Aware Rate Selection CARS algorithm. The proposed algorithm makes use of context information to systematically address some challenges such as, the rapid variations of the link quality caused by fading and mobility at vehicular speeds, and the transmission rate must adapt fast in order to be effective, during infrequent and bursty transmission.

The experimental evaluation in real outdoor vehicular environments with different mobility scenarios have showed the CARS adapts to changing link conditions at high vehicular speeds faster than existing rate-adaptation algorithms. The proposed scheme has achieved significantly higher throughput, up to $79 \%$, in all the tested scenarios, and is robust to packet loss due to collisions, improving the throughput by up to $256 \%$ in the presence of hidden stations.

Additionally, the researchers have showed another rate adaptation algorithm which underutilizes wireless link capacity in vehicular environments[26]. They have introduced an adaptive context aware rate selection (ACARS) algorithm that adapts to wireless and mobile channels with both path loss and shadowing effect. (ACARS) algorithm gave more realistic results and improvements compared to other algorithms.

New context-aware and Location-Based Service Discovery Protocols for VANETs (LocVSDP [EBLocVSDP]) have been proposed and their variant (NaiveLocVSDP)[27]. The proposed protocols relies on a cluster-based infrastructure and offers a scalable framework for the discovery of time-sensitive and location-based services in VANETs. Furthermore, the
LocVSDPs are integrated into the network layer and use channel diversity to improve service discovery efficiency. The simulation results have indicated that the proposed protocols shows a gain of $20 \%$ in terms of success rate. LocVSDPs uses at least $90 \%$ less bandwidth than VITP, and their average response time is at least $10 \%$ lower than VITP for successful query transactions.

The researchers in[28] have evaluated the performance of existing rate algorithms compared with context Aware Rate selection algorithm ( ACARS ) and how they can be applied to road safety and improve the network control and power management. The results have showed the proposed algorithm is able to minimize the power consumption for vehicles, because of its robustness among existing rate algorithms by the integration of power gain into the rate algorithm. The results have also showed the proposed algorithm can work well with the application of DSRC for road safety purposes. Additionally, there are some of benefits: reducing network congestion and improving the quality of service, collision avoidance, and road safety application.

By considering the context and critical services all over the area of interest, context-aware path protocol has been proposed [29]. The proposed protocol supports drivers with flexibility of considering the conditions of travelled road segments for decreasing the traffic congestion situation in downtown areas. It can be concluded from the experimental results that the percentage of passing through special road segments is reduced by $40 \%$ compared to previous congestion avoidance protocols.

To address the high mobility and frequent disconnection as challenges in VANETs, a collaborative $\mathrm{P} 2 \mathrm{P}$ information propagation scheme has been proposed[30]. A collaborative P2P contains of algorithms for data access/ dissemination/ and suggestion. This scheme aims to enhance the reliability of vehicle which addressed the broadcast storm problem when the irrelevant information may be sent to the vehicles. There is another an enhancement in the proposed scheme: connectivity ratio, medium usage ratio, and a reduction in extra messages generated along with improvement in optimal allocated rate .

Information dissemination over VANETs has major challenges such as, requiring large amount of bandwidth as the number of vehicles increases, and avoiding redundancy information generated by sharing data to neighbour nodes. A novel context aware data aggregation technique (CADA)[31] is proposed in VANETs which takes into account the context information before performing aggregation and dissemination. The simulations have been performed by using NS-3 and the results have showed there is $60 \%$ reduction in the required bandwidth for periodic data dissemination.

Context-aware driver's behaviour detection is a proposed system in VANETs that has been introduced[32] as a novel On Board Unit (OBU) architecture. The proposed system checks accurately and proactively four types of driving behaviour while driving: normal, 
fatigued, drunken and reckless driving. The context regarding the driver, the vehicle and the environment are taking into account. Then, the system will alert the driver and other vehicles on the road by operating in vehicle alarms and advising corrective action.

Furthermore, a novel Dynamic Bayesian Network $(\mathrm{DBN})$ framework has been introduced to perform reasoning about uncertainty and to infer the behaviour of drivers by collecting information. The results of this research have showed including more than one context data when concluding behaviour guarantees the detection of specific behaviours. Contextual reasoning and adaptation are possible as fundamental to trustworthy interactions among VANETs agents.

The researchers have been motivated[33] to integrate context information in a trustworthy management of VANETs. They have given a VANETs' architecture in which the agents, such as GTA, CA, and ERSU that are not part of VANETs. The merits of the proposed approach has been evaluated against the security attacks which are discussed by many researchers. The VANETs' architectural features can enable safety of the vehicles, improve traffic flow, and successfully survive different types of security attacks on the network.

A context-aware routing protocol is proposed for hovering information [5] which remains available in mobile nodes rather than in fixed infrastructure. The presented protocol uses the context of vehicles to make routing decisions. Road probability model is presented to calculate the probability of content to be disseminated on the roads. This study shows efficient results in terms of routing overhead and information availability. The presented protocol reduces the overhead by using an effective routing technique rather than using broadcast. A context-aware routing protocol is designed for both urban as well as highway scenarios.

To establish a fundamental solution to avoiding crashes from incidents and to create a safer environment, a context-aware pre-crash collision prediction system has been developed [34]. The proposed system uses a Dynamic Bayesian Network as a reasoning model to predict crash likelihood and severity level, whether any crash will be fatal, serious, or slight. The experiments have showed a highly accuracy in detecting the likelihood and severity of crashes, particularly when compared with the analysis of the contributory data of the DfT.

In [35], the researchers have developed a robust and reliable mechanism to support the positioning requirement of ITS services. This has been achieved by developing a high integrity GPS monitoring algorithm with the consideration of speed and based on the concept of context-awareness which can be applied with real time ITS services. Three phases of integrity are checked: (1) positioning integrity, (2) speed integrity, and (3) map matching integrity. Each phase uses different techniques to examine consistency of the GPS information. The results have showed the proposed algorithm has capability to support various types of location-based ITS services.
In [36], an effective on-street parking system has been proposed which helps to address and decrease a number of traffic congestion concerns relating to drivers' difficulties in locating an appropriate parking space. There is an increasing evidence considering that drivers spend a disproportionate time trying to locate a suitable parking space for their vehicle. So, any system can reduce this surely to be welcomed. The proposed system has offered to reduce congestion related stress and improve the driver experience by reducing the time it takes to identify and utilize a parking space that is available.

As mentioned above, context-aware systems provide information and services to users depending on the environmental contextual information.

\section{Video transmission over VANET}

Recently, both mobile and multimedia communications have experienced unequalled rapid growth and commercial success. However, transmitting multimedia flows over wireless ad-hoc networks remains an extremely challenging issue due to the limited battery lifetime of the wireless nodes.

In particular, video transmission over vehicular adhoc networks has been considered as a big issue and difficult task because of some challenges. For example, the huge enrichment by the video data of the quality of experience recognized by the driver and knowledge of the environment in many applications (emergency video calls, road traffic conditions, tourist information, etc). Furthermore, due to the strict Quality of Service requirements of video transmission[9].

Research studies in vehicular ad-hoc networks communication is playing a vital role in the future safety and ease of our roads. Whereas, the major goal of VANETs is the improvement and development of the driver safety and to reduce the rate of traffic death accidents and injuries by implementing collision avoidance and warning systems. Furthermore, VANETs can relieve traffic congestion by providing a driver with live routes that avoid road hazards and bottleneck areas. Thus, video traffic is playing a vital role in VANETs, because it supports navigation safety and gives drivers a precise and clear image of the traffic conditions when necessary. For fast response from users or drivers, visual images help in reacting quickly when required. However, video traffic is still considered a critical challenge which is the worst case that can be transmitted over the networks[9, 37-40].

There are many studies proposed in VOIP based video streaming applications with different routing protocols. The researchers have majorly contributed in this area by studying the effect of routing protocols to achieve robustness and quality of service of VOIP transmissions using different methodologies. These studies can be mentioned as follow:

The researchers [9] were looking forward the quality of service that allows the evaluation of the performance of 
a streaming video over VANETs especially in real time. Accordingly, several scenarios and multiple cases have been studied by measuring the delay, the jitter and the packets loss and using 802.11g. The faced problems were the time of transmission (real time) and the jitter. The experimental and theoretical studies showed that traffic and flow data corresponding to the IP-video format followed certain distributions that study have considered in the adopted models.

To enhance the dissemination of live video flows on multimedia highway VANETs with QoE (Quality of Experience) analysis, an application framework and DBD (Distributed Beaconless Dissemination) have been introduced[38]. The main contributions in this work was concluded in improving the performance of the IEEE 802.11p MAC layer. This work solved the Spurious Forwarding (SF) problem while increasing the packet delivery ratio and reducing the forwarding/destination delay. The simulation results showed that the DBD increased the SSIM of received videos in about $30 \%$ for scenarios with $100 \mathrm{veh} / \mathrm{km}$.

A QoE-based routing protocol for video transmission over VANETs has been proposed with respect of the mean opinion score (MOS) for path selection[39]. The novelty of this proposed mechanism comes from the fact that it combined QoE and OLSR protocols to guarantee a good video quality for users in VANETs. The simulation results highlighted how the proposed scheme improves the performance of video transmission applications in VANETs.

In [41], they have proposed a novel scheme for multiple description coding video streams transmission over VANETs through multiple paths. First, the video stream is divided into two redundancy description. Then, a clustering-based multiple paths algorithm is applied. For the purpose of improving network efficiency and reducing bandwidth consumption, the peer-to-peer scheme is applied in the data transmission between different layers at the same time. The simulation results have showed the effectiveness of the proposed scheme for more error resilient video transmission over ad hoc networks with high packet error rate.

Collaborative SVC (scalable video coding) video streaming techniques in VANETs have been introduced[42] by using V2V communications based on grouping vehicles into collaborative clusters. LTE is used to transmit the video to cluster heads. Additionally, the IEEE $802.11 \mathrm{p}$ is used for the communication between vehicles. The proposed techniques are extended to the V2I communication scenario. The simulation results have presented, analysed and demonstrated that significant enhancements in QoS and QoE metrics compared to the traditional non collaborative scenarios.

A video dissemination protocol for VANETs (VoV) with diverse traffic conditions has been proposed [43]. The proposed protocol utilizes a rate control mechanism, which makes this solution adaptable to the perceived network data traffic condition. In particular, the behaviour of all protocols is estimated under the real city scenarios.
The results have showed the increasing in reliability and decreasing in overhead of this proposed solution.

The researchers in [44] have discussed and used both qualitative and quantitative comparisons of number of selected approaches to study the effect of network coding on video dissemination protocols. Then, they have proposed hybrid video dissemination protocol which outperforms other protocols in term of delivery ratio and complies with other quality-of-service requirements for video broadcasting over vehicular environments.

A general study on the use of redundancy through Erasure Coding and Network Coding in both video unicast and video broadcast in VANETs has been provided [3]. The study has discussed novel approaches, investigated each strategy, and designed novel solutions then compared their performance. The proposed approach increases the overall video quality without leading to an excessive overhead nor to a substantial decrease in the receiving rate of unique video content.

A mathematical model has been conducted [45] in a framework applied to routing protocols in VANETs (AODV, DSR, and OLSR). Vehicle Rewarding for Video Transmission over VANETs using Real Neighbourhood and Relative Velocity (RNRV) has been developed for optimizing video transmission over VANET. The main goal of this work has been to maximize the VANETs' lifetime and to minimize the routing overhead of the network. The results have showed the number of real neighbours is critically influenced by the factors of relative velocity and distance of vehicles within the transmission range of the rewarded vehicle. Additionally, OLSR outperforms others in both network delay and throughput. Also OLSR has the lowest delay in all situations and levels of mobility and traffic, and that is useful for limiting the capacity of the environment using data streaming.

Video streaming applications over VANETs have been analysed[4] on applying (AODV) and (DYMO) in different traffic scenarios like varying node densities, node velocities and pause times. In VANETs, nodes join and leave the network quite frequently at high mobility resulting in route failures. A comparative analysis, that has been done on application layer metrics in order to further authenticate that VOIP traffic, shows better performance with AODV in terms of jitter, talking time and average mean opinion score (MOS). VOIP applications can be implemented with other routing protocols like LAR( Location aided Routing) and DSR (Dynamic Source Routing) to see the advantages of one over another.

Evaluation the quality of video transmitted over VANET has been analysed [46] by using models of QoS performance. They are the frame loss ratio, PSNR and connectivity probability, among popular routing protocols such as DSDV, AODV and GPSR with an integrated VANET test bed. The results of test have showed the position-based protocol is more suitable for video transmission over VANETs than other kind of protocols 
and it has also showed the scalability of different protocols.

An Optimal Scheduling Algorithm (OSA) has been proposed [47] for scalable video coding (SVC) and video streaming in highway VANETs. The extensive simulations have been conducted to evaluate the performance of OSA. The results have demonstrated that OSA outperforms all the competing schemes (Greedy scheme, the Average scheme and the Adaptive scheme) over variant density and velocity highway VANETs.

A selective Rebroadcaster selection mechanism (ReViV) has been proposed for Video streaming in VANETs [48]. The proposed mechanism selects a group of strategic broadcasting nodes, rather than all traditional node broadcasting mechanisms. For that purpose, the vehicles are ranked based on a new centrality metric called dissemination capacity DC (v) that they have introduced. This metric is inspired from node centrality metrics of Social Network Analysis (SNA). Through the simulations, the proposed mechanism has been compared with the multichannel vehicular communication standard IEEE 1609.4 and another pioneering video streaming mechanism over VANETs. The performance evaluation has showed that the proposed mechanism outperforms the above mentioned mechanisms by providing higher video delivery ratio, lower end-to-end transmission delay and lower frame loss ratio in both fully and intermittently connected networks[49].

\section{Conclusion}

Context-aware vehicular ad-hoc network is a novel field that offers enhancement solutions by using context-aware technique to address the challenges of VANETs and guarantee the stability of topology through different high mobility of nodes. In this paper, we investigated the common issues that related to vehicular ad-hoc networks such as characteristics, applications, current challenges and ad-hoc based routing related to VANETs. These issues have been taken into consideration to enable the implementation of highly efficient communication protocols and ad-hoc based routing over VANETs. Moreover, we reviewed the most important relevant studies that included context-aware issue as a new technique by using contextual information in video transmission to overcome VANETs challenges.

\section{References}

[1] T. A. Binjammaz, A. H. Al-Bayatti, and A. AlHargan, "Context-aware GPS integrity monitoring for intelligent transport systems," Journal of Traffic and Transportation Engineering (English Edition), vol. 3, pp. 1-15, 2016.
[2] E. C. Eze, S. Zhang, and E. Liu, "Vehicular ad hoc networks (VANETs): Current state, challenges, potentials and way forward," in Automation and Computing (ICAC), 2014 20th International Conference on, 2014, pp. 176-181.

[3] C. Rezende, A. Boukerche, M. Almulla, and A. A. Loureiro, "The selective use of redundancy for video streaming over Vehicular Ad Hoc Networks," Computer Networks, vol. 81, pp. 43-62, 2015.

[4] P. Sharma, A. Kaul, and M. Garg, "Performance analysis of video streaming applications over VANETs," International Journal of Computer Applications, vol. 112, 2015.

[5] Z. Amjad, W.-C. Song, and K.-J. Ahn, "Context-Aware Routing for hovering information in Vehicular Ad-Hoc Networks," in Network Operations and Management Symposium (APNOMS), 2016 18th AsiaPacific, 2016, pp. 1-6.

[6] E. G. Singh, "Overview of Challenges in VANET," 2016.

[7] S. Al-Sultan, M. M. Al-Doori, A. H. Al-Bayatti, and $\mathrm{H}$. Zedan, "A comprehensive survey on vehicular ad hoc network," Journal of network and computer applications, vol. 37, pp. 380-392, 2014.

[8] W. Liang, Z. Li, H. Zhang, S. Wang, and R. Bie, "Vehicular ad hoc networks: architectures, research issues, methodologies, challenges, and trends," International Journal of Distributed Sensor Networks, vol. 11, p. 745303, 2015.

[9] A. Ennaciri, M. Erritali, M. Mabrouki, and J. Bengourram, "Performance analysis of Streaming Video over Vehicular ad-hoc," in Computer Graphics, Imaging and Visualization (CGiV), 2016 13th International Conference on, 2016, pp. 375380.

[10] D. Hardeep and R. Malhotra, "Evaluation of Routing Protocols for Adhoc Network in Vehicular Environment," International Journal of Engineering Science, vol. 7709, 2016.

[11] P. T. Mahida, R. Patel, P. Patel, and S. Mody, "Performance evaluation of dynamic and static sensor node in wireless sensor 
network," Indian J. Comput. Sci. and Eng, vol. 4, pp. 23-28, 2013.

[12] A. Shaheen, A. Gaamel, and A. Bahaj, "Comparison and Analysis Study between AODV and DSR Routing Protocols in VANET with IEEE 802.11 b," Journal of Ubiquitous Systems \& Pervasive Networks, vol. 7, pp. 07-12, 2016.

[13] N. M. Mittal and S. Choudhary, "Comparative study of simulators for vehicular ad-hoc networks (vanets)," International Journal of Emerging Technology and Advanced Engineering Website: www. ijetae. com (ISSN 22502459, ISO 9001: 2008 Certified Journal, Volume 4, Issue 4, 2014.

[14] A. K. Pandey, "Simulation of traffic movement in vanet using sumo," 2013.

[15] P. Ranjan and K. K. Ahirwar, "Comparative study of vanet and manet routing protocols," in Proc. of the International Conference on Advanced Computing and Communication Technologies (ACCT 2011), 2011, pp. 517-523.

[16] A. Dua, N. Kumar, and S. Bawa, "A systematic review on routing protocols for vehicular ad hoc networks," Vehicular Communications, vol. 1, pp. 33-52, 2014.

[17] A. B. Chagas and C. A. Ferraz, "Smart Vehicles for Smarter Cities: Context-Aware V2X to Improve Qol," 2017.

[18] V. Alagar and K. Wan, "Context-Aware Trust-Based Management of Vehicular AdHoc Networks (VANETs)," in Ubiquitous Intelligence and Computing and 2015 IEEE 12th Intl Conf on Autonomic and Trusted Computing and 2015 IEEE 15th Int Conf on Scalable Computing and Communications and Its Associated Workshops (UIC-ATCScalCom), 2015 IEEE 12th Intl Conf on, 2015, pp. 255-261.

[19] M. Z. Aswad, "Context aware pre-crash system for vehicular ad hoc networks using dynamic bayesian model," 2014.

[20] B. Paul, M. Ibrahim, M. Bikas, and A. Naser, "VANET routing protocols: Pros and cons," arXiv preprint arXiv:1204.1201, 2012.

[21] S. Yang, R. He, Y. Wang, S. Li, and B. Lin, "OPNET-based modeling and simulations on routing protocols in VANETs with IEEE
$802.11 \mathrm{p}, "$ in Systems and Informatics (ICSAI), 2014 2nd International Conference on, 2014, pp. 536-541.

[22] S. M. Vishal Kumar, Narottam Chand, "Applications of VANETs: Present \& Future," Communications and Network, vol. 5, pp. 12-15, 2013.

[23] F. Cunha, L. Villas, A. Boukerche, G. Maia, A. Viana, R. A. Mini, et al., "Data communication in VANETs: Protocols, applications and challenges," Ad Hoc Networks, vol. 44, pp. 90-103, 2016.

[24] C. Casetti, M. Cesana, I. Filippini, G. Dán, and I. Marsh, "Context-aware information dissemination in vehicular networks," in Next Generation Internet (NGI), 2011 7th EURO-NGI Conference on, 2011, pp. 1-2.

[25] S. Kukliński and G. Wolny, "CARAVAN: a context-aware architecture for VANET," in Mobile Ad-Hoc Networks: Applications, ed: InTech, 2011.

[26] P. Shankar, T. Nadeem, J. Rosca, and L. Iftode, "Cars: Context-aware rate selection for vehicular networks," in Network Protocols, 2008. ICNP 2008. IEEE International Conference on, 2008, pp. 1-12.

[27] K. Abrougui, A. Boukerche, and R. W. N. Pazzi, "Design and evaluation of contextaware and location-based service discovery protocols for vehicular networks," IEEE Transactions on Intelligent Transportation Systems, vol. 12, pp. 717-735, 2011.

[28] K. S. Nwizege, J. He, K. S. Kim, and P. Igic, "Performance evaluation of adaptive context aware rate selection algorithm (acars) for road safety applications in vehicular network," in Modelling Symposium (EMS), 2013 European, 2013, pp. 640-646.

[29] A. B. Maram Bani Younes, "A Performance Evaluation of a Context-Aware Path Recommendation Protocol for Vehicular Adhoc Networks," Ad Hoc and Sensor Networking Symposium, 2013.

[30] N. Kumar, R. Iqbal, A. E. James, and A. Dua, "Collaborative P2P context-aware information propagation in vehicular ad hoc networks," in Computer Supported Cooperative Work in Design (CSCWD), 2015 
IEEE 19th International Conference on, 2015, pp. 292-296.

[31] W. C. S. Zubair Amjad*, Khi Jung Ahn, Muhammad Shoaib, "Context Aware Data Aggregation in Vehicular Ad-hoc Networks," International Workshop on Platforms and Applications for Smart Cities (PASC), 2016.

[32] S. J. Al-Sultan, "Context Aware Drivers' Behaviour Detection System for VANET," PhD, Software Technology Research Lab oratory, De Montfort University, Leicester United Kingdom, 2013.

[33] K. W. n. Vangalur Alagar, "Context-aware Trust-based Management of ( VANETs )," IEEE Transactions on Intelligent Transportation Systems, 2015.

[34] M. Z. A. R. Aswad, "CONTEXT AWARE PRECRASH SYSTEM FOR VEHICULAR AD HOC NETWORKS USING DYNAMIC BAYESIAN MODEL," PhD, Software Technology Research Laboratory, De Montfort University, Leicester - United Kingdom.

[35] T. A. Binjammaz, "Context-aware GPS Integrity Monitoring for Intelligent Transport Systems (ITS)," PhD, Software Technology Research Laboratory, De Montfort University, United Kingdom, 2015.

[36] A. A. Alhammad, "Context-Aware Aided Parking Solutions Based on VANET," PhD, Software Technology Research Laboratory, De Montfort University, Leicester - United Kingdom, 2014.

[37] O. A. Wahab, H. Otrok, and A. Mourad, "VANET QoS-OLSR: QoS-based clustering protocol for vehicular ad hoc networks," Computer Communications, vol. 36, pp. 1422-1435, 2013.

[38] M. De Felice, E. Cerqueira, A. Melo, M. Gerla, F. Cuomo, and A. Baiocchi, "A distributed beaconless routing protocol for real-time video dissemination in multimedia VANETs," Computer Communications, vol. 58, pp. 40-52, 2015.

[39] T. A. Q. Pham, K. Piamrat, and C. Viho, "QoE-aware routing for video streaming over VANETs," in Vehicular Technology Conference (VTC Fall), 2014 IEEE 80th, 2014, pp. 1-5.

[40] M. Chaqfeh, A. Lakas, and I. Jawhar, "A survey on data dissemination in vehicular ad hoc networks," Vehicular Communications, vol. 1, pp. 214-225, 2014.

[41] Y. Chen, S. Yu, J. Zhou, and J. Fan, "Video transmission over ad hoc networks using multiple description coding and clusteringbased multiple paths," in Software Engineering, Artificial Intelligence, Networking, and Parallel/Distributed Computing, 2007. SNPD 2007. Eighth ACIS International Conference on, 2007, pp. 792797.

[42] E. Yaacoub, F. Filali, and A. Abu-Dayya, "QoE enhancement of SVC video streaming over vehicular networks using cooperative LTE/802.11 p communications," IEEE Journal of Selected Topics in Signal Processing, vol. 9, pp. 37-49, 2015.

[43] G. Maia, L. A. Villas, A. C. Viana, A. L. Aquino, A. Boukerche, and A. A. Loureiro, "A rate control video dissemination solution for extremely dynamic vehicular ad hoc networks," Performance Evaluation, vol. 87, pp. 3-18, 2015.

[44] F. Naeimipoor, "Video streaming and multimedia broadcasting over vehicular Ad Hoc networks," Université d'Ottawa/University of Ottawa, 2013.

[45] W. S. M. Yousef, M. R. H. ARSHAD, and A. Zahary, "VEHICLE REWARDING FOR VIDEO TRANSMISSION OVER VANETS USING REAL NEIGHBORHOOD AND RELATIVE VELOCITY (RNRV)," Journal of Theoretical \& Applied Information Technology, vol. 95, 2017.

[46] S. Xu, P. Guo, B. Xu, and H. Zhou, "QoS evaluation of VANET routing protocols," Journal of Networks, vol. 8, p. 132, 2013.

[47] R. An, Z. Liu, and Y. Ji, "Video streaming for highway vanet using scalable video coding," in Vehicular Technology Conference (VTC Fall), 2014 IEEE 80th, 2014, pp. 1-5.

[48] A. Bradai and T. Ahmed, "ReViV: Selective rebroadcast mechanism for video streaming over VANET," in Vehicular Technology Conference (VTC Spring), 2014 IEEE 79th, 2014, pp. 1-6.

[49] J. Yang and Z. Fei, "Broadcasting with prediction and selective forwarding in vehicular networks," International journal of distributed sensor networks, vol. 9, p. 309041, 2013. 